\title{
Coniferyl Aldehyde Inhibits the Inflammatory Effects of Leptomeningeal Cells by Suppressing the JAK2 Signaling
}

\author{
Yao Wang $\left(\mathbb{D},{ }^{1}\right.$ Yajun Gao $\mathbb{D}^{2},{ }^{2}$ Xue Li, ${ }^{1}$ Xiaolin Sun, ${ }^{1}$ Zhanqi Wang, ${ }^{1}$ Hanchi Wang, ${ }^{1}$ Ran Nie, \\ Weixian Yu (1), and Yanmin Zhou (1) \\ ${ }^{1}$ Department of Oral Implantology, School and Hospital of Stomatology, Jilin University, Changchun 130021, China \\ ${ }^{2}$ VIP Integrated Department, School and Hospital of Stomatology, Jilin University, Changchun 130021, China \\ ${ }^{3}$ Jilin Provincial Key Laboratory of Tooth Development and Bone Remodeling, School and Hospital of Stomatology, Jilin University, \\ Changchun 130021, China
}

Correspondence should be addressed to Weixian Yu; ywx@jlu.edu.cn and Yanmin Zhou; zhouym1962@126.com

Received 29 January 2020; Revised 29 June 2020; Accepted 11 August 2020; Published 15 September 2020

Academic Editor: Mauro S. Oliveira

Copyright (c) 2020 Yao Wang et al. This is an open access article distributed under the Creative Commons Attribution License, which permits unrestricted use, distribution, and reproduction in any medium, provided the original work is properly cited.

Background. The brain is in many ways an immunologically and pharmacologically privileged site because of the blood-brain barrier (BBB). But for chronic peripheral inflammation, inflammatory signals can be transmitted from the peripheral system into the central nervous system (CNS) through multiple channels and result in neuroinflammation. Leptomeningeal cells that form the BBB can trigger one signaling pathway by releasing cytokines to transmit inflammatory signals. Besides, the Janus kinase (JAK) family may have a certain function in the activation of leptomeninges. In the present study, we try to use coniferyl aldehyde (CA), a natural anti-inflammatory phenolic compound, to inhibit this inflammatory process and elucidate the underlying molecular mechanisms. Results. Secretion of proinflammatory cytokines (TNF- $\alpha$, IL-1 $\beta$, and IL-6) significantly increased after incubation with P. gingivalis. Moreover, TNF- $\alpha$, IL-1 $\beta$, and IL-6 levels were upregulated, and the JAK2 signaling was enhanced in leptomeningeal cells in a conditioned medium from activated macrophages, which leads to the immune response in microglia. However, this inflammatory effect of leptomeningeal cells was reversed by CA administration, accompanied by the decreased immune response in microglia. The western blot assay revealed that JAK2 phosphorylation was suppressed in leptomeningeal cells treated with CA. Conclusions. This study demonstrates that activated macrophages by $P$. gingivalis markedly induce the release of proinflammatory cytokines (TNF- $\alpha$, IL- $1 \beta$, and IL- 6 ) from leptomeningeal cells, thereby activating the JAK2 signaling pathway and subsequently enhancing immune responses in microglia in the CNS. CA effectively inhibits the inflammatory effect of leptomeningeal cells via suppressing the JAK2 signaling pathway.

\section{Introduction}

Periodontitis (PD) is a chronic inflammatory disease caused by the presence of a bacterial biofilm called dental plaque, which has an impact on the periodontal ligaments and bone surrounding teeth [1]. It is estimated that severe PD afflicts $\sim 8 \%$ of the USA adults [2]. $P$. gingivalis is an anaerobic bacteria that inhabits the oral cavity and is considered to be the most common pathogenic bacterium in patients with chronic PD [3]. Evidences show that the polymicrobial synergy involving $P$. gingivalis in the oral cavity of $\mathrm{PD}$ patients causes host immune responses against $P$. gingivalis infections at systemic sites [4], followed by releases of inflammatory mediators into the blood circulation [5]. This indicates an underlying association between PD and neuroinflammation $[5,6]$. However, there is still dispute over how inflammatory signals enter central nervous system (CNS) through bloodbrain barrier (BBB).

Some researchers $[7,8]$ found that leptomeningeal cells which constitute the $\mathrm{BBB}$ may transduce inflammatory signals to inflammatory cells in the CNS in response to lipopolysaccharide (LPS) of $P$. gingivalis. The leptomeninges were always thought to be in the front line against infections to defend the CNS $[9,10]$, whereas meninges serve not only as a physical defense against infectious molecules, but also involve in signal transmission [11], molecule transport [12], 
stem cell incubation [11], and the immune responses in brain-resident inflammatory cells in the CNS [13]. Further evidence [14] shows that many substances, receptors, and cytokines, such as LPS, interleukin-1 $\beta$ (IL- $1 \beta)$, interleukin6 (IL-6), cyclooxygenase (COX)-2, I $\kappa \mathrm{B} \alpha$, tumor necrosis factor $\alpha(\mathrm{TNF}-\alpha)$, and prostaglandin (PG), pronouncedly enhance secretion of more inflammatory factors from leptomeningeal cells. That means cytokines coming from leptomeningeal cells have been activated in the CNS even before the imminent entry of $P$. gingivalis into the brain. However, the exact mechanism has not been fully explored.

The immune response of CNS called neuroinflammation is characterized by the presence of activated microglia which has the unique capability to phagocytose toxic products, release cytotoxic factors, and behave as antigen-presenting cells [15-17]. Neuroinflammation is a common pathological process of multiple neurological diseases, such as neurodegenerative dementias $[18,19]$, intracerebral hemorrhage (ICH) $[20,21]$, and severe traumatic brain injury (sTBI) [22]. Chronic focal inflammation is considered to induce neuroinflammation through leptomeningeal cells. Epidemiological evidences show that early administration of nonsteroidal anti-inflammatory drugs (NSAIDs) reduces the risk of Alzheimer's disease (AD) [23].

As an important class of kinases in the human body, the Janus kinase (JAK) family is expressed in almost all cells. JAK2 as the most important phenotype and the most conserved isoform of the JAK family involves in various pathological processes. After activation of cell surface receptor induced by cytokines or LPS, JAK2 proteins will be recruited and phosphorylated, leading to the phosphorylation of the downstream STAT3. Phosphorylated STAT3 homodimerizes and translocates to the nucleus where it binds to the promoter of its target genes and activates their transcription to participate in fibrosis, angiogenesis, and inflammation in numerous diseases [24]. In our study, AG490 (a JAK2specific inhibitor) was employed, aiming to figure out whether JAK2 involves in inflammation responses in leptomeningeal cells induced by cytokines from macrophages and whether it is a therapeutic target of neurodegenerative diseases.

Besides, we try to find out a new anti-inflammatory drug for the treatment of neuroinflammation and the resultant neurodegenerative diseases. Coniferyl aldehyde (CA) is the component of a natural non-toxic phenolic compound extracted from dietary and medicinal plants. It has always been used in the wine manufacture until recently its medical implication has been discovered [25-27].

The objectives of this study were to validate that leptomeningeal cells transmit inflammatory signals from bloodborne immune cells to brain-resident microglia and to expound the underlying mechanisms and drug intervention for the first time. We hypothesized that (1) P. gingivalis mediated the immune responses in macrophages; (2) secretions of macrophages elevated inflammatory levels of cytokines released from leptomeningeal cells; (3) the cytokines from leptomeningeal cells initiated inflammatory microglial responses; and (4) these inflammatory processes could be inhibited by CA via suppressing the JAK2/STAT signaling pathway.

\section{Materials and Methods}

2.1. Cell-Line Culture. The macrophage cell line RAW264.7 was purchased from the ATCC and the mouse microglia BV2 cell line from BeNa (Beijing, China). The cells were cultured in Dulbecco's Modified Eagle Medium (DMEM, Hyclone, USA) supplemented with $10 \%$ fetal bovine serum (FBS, Biological Industries, Israel Beit-Haemek), penicillin $\mathrm{G}$, and streptomycin (100 $\mu \mathrm{g} / \mathrm{mL}$, Hyclone, USA). The cells were incubated at $37^{\circ} \mathrm{C}$ in a humidified, $5 \% \mathrm{CO}_{2}$ atmosphere.

2.2. Leptomeningeal Cell Culture. Preparations of leptomeningeal cell culture were described elsewhere $[7,28]$. The brains of 3-day-old C57black/6N mice were collected and soaked in an ice-cold phosphate buffer saline (PBS). Leptomeningeal tissues were collected, plated on a poly-D-lysinecoated culture flask, and incubated in DMEM (Hyclone, USA) containing 10\% FBS (Biological Industries, Israel Beit-Haemek), penicillin G, and streptomycin $(100 \mu \mathrm{g} / \mathrm{mL}$, Hyclone, USA). After 7 days, the culture flask was strongly shaken and washed with a sterile, isotonic $\mathrm{Ca}^{2+} / \mathrm{Mg}^{2+}$-free buffer ( $\mathrm{pH} 7.0$ ), which consisted of $137 \mathrm{mM} \mathrm{NaCl}, 5 \mathrm{mM}$ $\mathrm{KCl}, 0.7 \mathrm{mM} \mathrm{KH} \mathrm{KO}_{4}, 25 \mathrm{mM}$ glucose, $59 \mathrm{mM}$ sucrose, $0.3 \%$ bovine serum albumin (BSA), and penicillin and streptomycin $(100 \mu \mathrm{g} / \mathrm{mL})$. This step could remove any contaminated cells such as neuronal and glial cells $[8,29]$.

2.3. Macrophage Conditioned Medium (MCM) Was Collected to Analyze Cytokines by an ELISA. Macrophages were placed into a $10 \mathrm{~cm}$ culture dish $\left(5 \times 10^{5}\right.$ cells/dish $)$. The cells were treated with $\mathrm{PBS}$, alive $P$. gingivalis, or $P$. gingivalis LPS $(5 \mu \mathrm{g} / \mathrm{mL})$. Another set of cells was preincubated with AG490 (Sigma, USA), a JAK2-specific inhibitor $(50 \mu \mathrm{g} / \mathrm{mL})$, for $12 \mathrm{~h}$ before treated with alive $P$. gingivalis. After $24 \mathrm{~h}$ of administration, supernatants of cultured macrophages passed through a filter to remove bacteria and cells. The levels of two classic cytokines in these supernatants were determined using an ELISA (R\&D Systems, Inc., Minneapolis, MN, USA). The experimental procedure was performed in accordance to the manufacturer's instructions. The MCM in the $P$. gingivalis group was collected and stored at $-20^{\circ} \mathrm{C}$ up to 2 weeks or $-80^{\circ} \mathrm{C}$ up to 3 months. On the other hand, alive $P$. gingivalis was added to complete medium and stored at $37^{\circ} \mathrm{C}$ in a humidified, $5 \% \mathrm{CO}_{2}$ atmosphere for $24 \mathrm{~h}$. It was then filtered, and the supernatant was marked as "bacteriafree culture broth filtrate (BF)". The two liquids above were both going to be used in the following experiment.

2.4. Leptomeningeal Cells Were Treated with MCM. Leptomeningeal cells were seeded in 6-well culture plates. The cells were incubated with complete medium, MCM, or BF that we had prepared in the previous experiment. Another set of cells was preincubated with AG490 before treated with MCM. The cells from all groups were incubated at $37^{\circ} \mathrm{C}$ for $6 \mathrm{~h}$, and the medium was renewed. After another $6 \mathrm{~h}$ of incubation, the new medium was filtered, and the supernatant was collected. Cytokines in the leptomeningeal-cell-conditioned mediums (LCMs) of all groups were also analyzed by an ELISA, using the same procedures as previously described. Besides, mRNA expression levels of the three inflammatory cytokines in 
TABLE 1: Sequences of the primers used in RT-qPCR assays.

\begin{tabular}{lc}
\hline Target genes & Sequence \\
\hline Actin- $\beta$ & $5^{\prime}$-CATCCGTAAAGACCTCTATGCCAAC-3' \\
& $5^{\prime}$-ATGGAGCCACCGATCCACA-3' \\
\hline TNF- $\alpha$ & $5^{\prime}$-ACTCCAGGCGGTGCCTATGT-3' \\
& $5^{\prime}$-GTGAGGGTCTGGGCCATAGAA-3' \\
\hline IL-6 & $5^{\prime}$-CCACTTCACAAGTCGGAGGCTTA-3' \\
\hline & $5^{\prime}$-CCAGTTTGGTAGCATCCATCATTTC-3' \\
IL-1 $\beta$ & $5^{\prime}$-TCCAGGATGAGGACATGAGCAC-3' \\
& $5^{\prime}$-GAACGTCACACACCAGCAGGTTA-3'
\end{tabular}

leptomeningeal cells were quantitated using real time quantitative polymerase chain reaction (RT-qPCR) analysis. Following the manufacturer's instructions, the total mRNA was extracted using a Hipure total mRNA Mini kit (Magen, China). A total of $1000 \mathrm{ng}$ of extracted RNA was reversely transcribed to cDNA using the Primescript RT reagent Kit with gDNA Eraser (Takara, Japan). The thermal cycling was set at $42^{\circ} \mathrm{C}$ for $2 \mathrm{~min}$, and then at $37^{\circ} \mathrm{C}$ for $15 \mathrm{~min}$, followed by $85^{\circ} \mathrm{C}$ for $5 \mathrm{~s}$. The cDNA was amplified in duplicate using the TB Green Premix Ex Taq II (Takara, Japan). The primer sequences used were listed in Table 1. Finally, all LCMs in MCM group, MCM+AG490 group, and BF group were collected and filtered. The supernatants were labeled as "LCM (MCM)," "LCM (MCM+AG490)," and "LCM (BF)," respectively, which were going to be used in the following experiment.

2.5. Microglia Were Treated with LCM. Microglia were treated with complete medium or LCM (MCM) or LCM $(\mathrm{MCM}+\mathrm{AG} 490)$ or LCM (BF) for $12 \mathrm{~h}$. Then, the medium was renewed to remove the cytokine environment. The cells together with the new medium were collected after another $12 \mathrm{~h}$ of incubation. Protein and mRNA expression levels of inflammatory factors were determined using an ELISA and RT-qPCR, respectively.

2.6. Cytotoxicity of CA Was Evaluated Using the CCK-8 Assay and Fluorescent Staining. Leptomeningeal cells were plated into 96-well plates $\left(1 \times 10^{4}\right.$ cells per well $)$ and 6 -well plates $\left(1 \times 10^{4}\right.$ cells per well). They were incubated with 1,5 , and $50 \mu \mathrm{M}$ CA and PBS for $12 \mathrm{~h}$. Leptomeningeal cells in 6-well plates were stained with FITC and DAPI to evaluate their morphological structure after CA administrations. The proliferation activity of the cell was determined using the cell-counting kit-8 (CCK-8) assay (Dojindo, Kumamoto, Japan). After $4 \mathrm{~h}$ of incubation with CCK-8, the OD value at $450 \mathrm{~nm}$ was measured by a microplate reader.

2.7. Inflammatory Responses of Leptomeningeal Cells after CA Administration Were Assessed Using RT-qPCR Analysis. Leptomeningeal cells pretreated with 1,5 , and $50 \mu \mathrm{M}$ CA for $12 \mathrm{~h}$ were cultured with MCM for another $12 \mathrm{~h}$. Subsequently, the cells were collected, and IL-6 mRNA expression levels were determined by RT-qPCR analysis. The supernatants of all groups were collected and labeled as "LCM (MCM)," "LCM (MCM+1 $\mu \mathrm{M}$ CA)," "LCM (MCM+5 $\mu \mathrm{M}$ CA)," and "LCM $(\mathrm{MCM}+50 \mu \mathrm{M} \mathrm{CA})$, , and microglia were incubated with these conditioned mediums at $37^{\circ} \mathrm{C}$ for $24 \mathrm{~h}$. The mRNA expression level of proinflammatory cytokine IL-6 was determined by RT-qPCR.

2.8. Phosphorylated JAK2 Levels in Leptomeningeal Cells after CA Administrations Were Analyzed Using Western Blot Assay. Leptomeningeal cells in the $\mathrm{MCM}+\mathrm{CA}$ group were preincubated with $50 \mu \mathrm{M}$ CA for $12 \mathrm{~h}$ before the medium was replaced with MCM. The cells in the MCM group were only incubated with MCM. The cells in the control group were not treated with any special drug. The expressions of phosphorylated JAK2 in the three groups were quantitated using western blot analysis. The anti-p-JAK2 antibody was provided by Sigma (Merck KGaA, Darmstadt, Germany) and the protein extraction kit by ABclonal (Wuhan, China).

2.9. Statistical Analysis. The data are represented as the means $\pm \operatorname{SEM}(n=3$, each); all assays were repeated three times with independent samples. The GraphPad Prism (GraphPad Software, San Diego, CA, USA) was used to perform statistical analyses. One-way ANOVA or Student's $t$-test was used for comparisons between groups. A value of $P<0.05$ was considered to indicate statistical significance.

\section{Results}

3.1. The Effect of P. gingivalis on the Inflammatory Factors from Macrophages. To validate the immune responses in macrophages, we detected the inflammatory levels of cytokines in the medium. The protein levels of two cytokines TNF- $\alpha$ and IL- 6 were significantly higher in the $P$. gingivalis group and the LPS group than those in the control group $(P<0.001)$ (Figure 1). Unexpectedly, these inflammatory cytokines showed distinct sensitivities to $P$. gingivalis and $P$. gingivalis LPS. The upregulation of TNF- $\alpha$ in macrophages treated with $P$. gingivalis was weaker than that in macrophages treated with $P$. gingivalis LPS. On the contrary, the upregulation of IL- 6 was stronger in those treated with P. gingivalis than those in macrophages treated with $P$. gingivalis LPS. TNF- $\alpha$ and IL-6 levels significantly decreased in the $P$. gingivalis+AG490 group. These results confirmed that $P$. gingivalis stimulated inflammatory reactions of macrophages, and this effect was partially reversed when JAK2 was suppressed.

\subsection{P. gingivalis Triggered Macrophage-Dependent Immune} Response in Leptomeningeal Cells. After the inflammatory reaction of macrophages had been evaluated in the previous experiment, we tried to explore the influence of macrophages on leptomeningeal cells. TNF- $\alpha$, IL- $1 \beta$, and IL- 6 mRNA expressions significantly increased in the MCM group compared to those in the control and negative control group (or the BF group) (Figure 2). Similar results were observed when we detected their protein levels using an ELISA. Surprisingly, protein levels of IL- $1 \beta$ were very small or even detected naught in some wells. There was no significant difference 


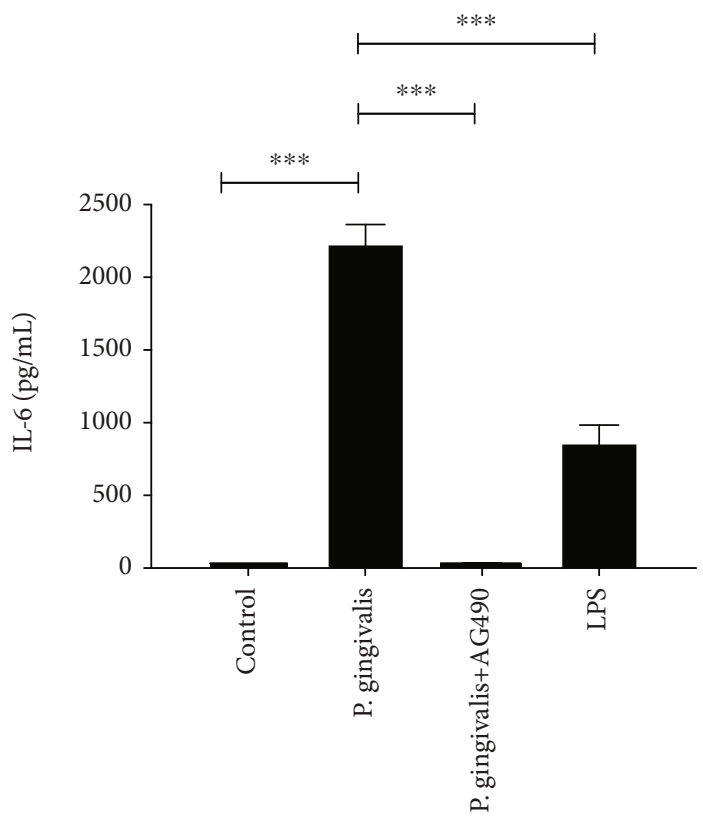

(a)

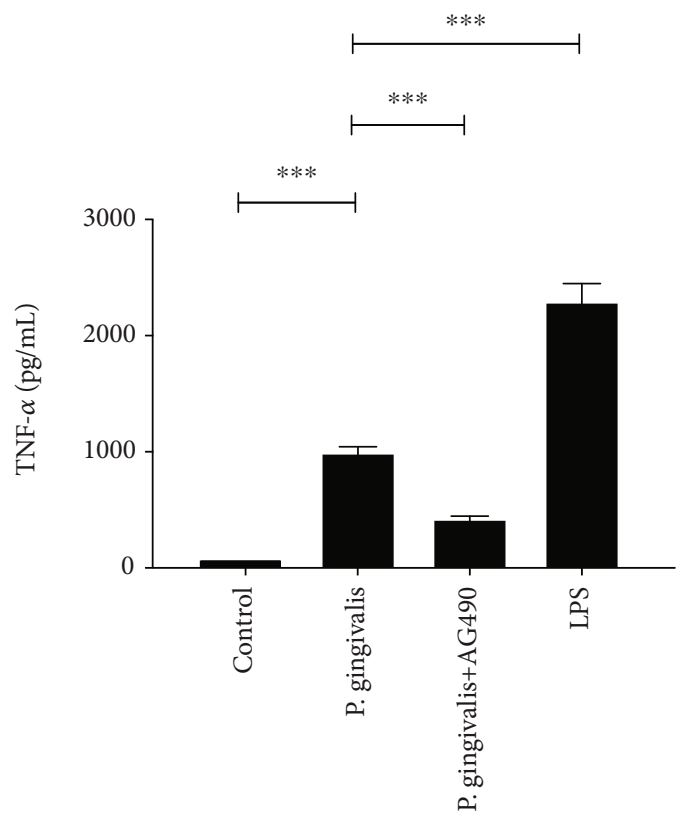

(b)

Figure 1: Inhibitory effects of AG490 on the secretion of proinflammatory cytokines by $P$. gingivalis-treated macrophages. (a, b) The mean concentrations of TNF- $\alpha$ and IL-6 in the culture medium of macrophages exposed to P. gingivalis or P. gingivalis LPS for $24 \mathrm{~h}$ were measured by ELISA. Each column and bar represent the mean \pm SEM $(n=3$, each). Asterisks indicate a statistically significantly difference from the value in corresponding group $(* * * P<0.001)$.

between all groups. For BF group, we found that its inflammatory stimulation was slight compared with the MCM group. This supported our hypothesis that macrophages rather than bacterial soluble metabolites triggered the inflammatory response in leptomeningeal cells.

On the other hand, AG490 $(50 \mu \mathrm{g} / \mathrm{mL})$ was further used to understand the role of JAK2 in the inflammatory response of leptomeningeal cells. The levels of the three cytokines in the MCM+AG490 group were lower than those in the MCM group.

3.3. The LCM (MCM) Induced Microglial to Express Proinflammatory Cytokines. In order to understand the effect of activated leptomeningeal cells on immune microglial responses, the major immune cells in the brain, we used LCMs to stimulate the microglia and observe its reaction in this step. It was found that LCM (MCM) triggered immune responses in microglia. And the stimulating effects of LCM $(\mathrm{MCM}+\mathrm{AG} 490)$ and LCM (BF) on microglia were weaker than the effect of LCM (MCM) (Figure 3). This meant the activity of inflammatory signals was inhibited with the presence of AG490 $(50 \mu \mathrm{g} / \mathrm{mL})$. Similarly, LCM (MCM) did not increase IL- $1 \beta$ protein levels in the medium even though its mRNA levels in the LCM (MCM) group were much higher than those in the control group.

3.4. Cytotoxicity of CA to Leptomeningeal Cells Was Negligible. In order to confirm the optimal dose of CA, which showed a potent anti-inflammation effect without affecting the cellular activity, the cytotoxicity of CA was assessed. After $24 \mathrm{~h}$ of $1,5,50 \mu \mathrm{M}$ CA treatment, immunofluorescence anal- ysis showed that all cells were long-fusiform shapes or irregular polygons, and the nuclei were round or oval. There were no significant changes in the morphology of the cells between groups under a microscope (Figure 4(a)). There was a nonsignificant difference in cell proliferative between CA groups and the control group (Figure 4(b)). This suggested that 1, 5, and $50 \mu \mathrm{M}$ CA treatment had negligible effects on the morphology and proliferation of leptomeningeal cells.

3.5. CA Suppressed the Inflammatory Reaction of Leptomeningeal Cells. The leptomeningeal cells incubated with MCM+CA showed a lower IL-6 mRNA level than those treated with MCM. As the concentration of CA increased, IL-6 mRNA levels decreased (Figure 5).

For microglia, there was a nonsignificant difference in the IL-6 mRNA level between the LCM (MCM) and LCM $(\mathrm{MCM}+1 \mu \mathrm{M} \mathrm{CA})$ groups. But IL-6 levels in the LCM $(\mathrm{MCM}+5 \mu \mathrm{M}$ CA) and $\mathrm{LCM}(\mathrm{MCM}+50 \mu \mathrm{M} \mathrm{CA})$ groups were both lower than the level in the LCM (MCM) group (Figure 6).

3.6. CA Partially Inhibited P-JAK2 Expressions in Leptomeningeal Cells Incubated with MCM. To identify the exact signaling pathway involving in the anti-inflammatory activities of CA, we assessed the phosphorylation level of JAK2 in the MCM+CA, MCM, and control groups using western blot assay. After $12 \mathrm{~h}$ of CA incubation, the p-JAK2/JAK2 level in the MCM+CA group was higher than that in the control group (Figure 7). However, the p-JAK2/JAK2 level significantly decreased in the $\mathrm{MCM}+\mathrm{CA}$ group 


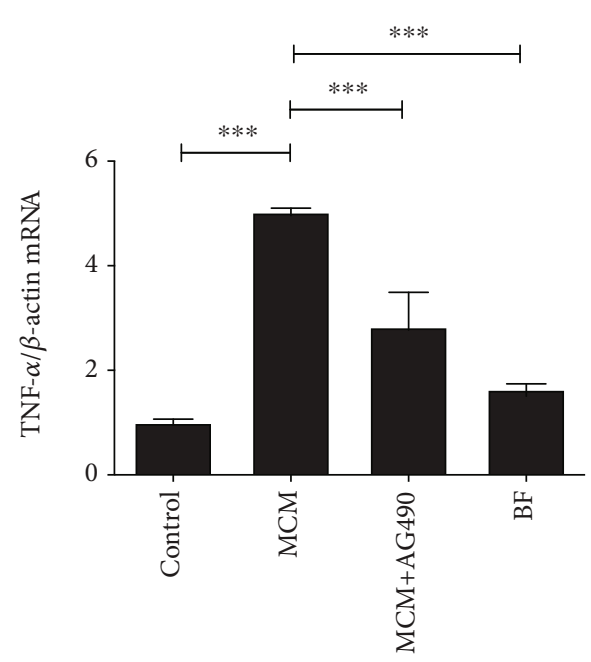

(a)

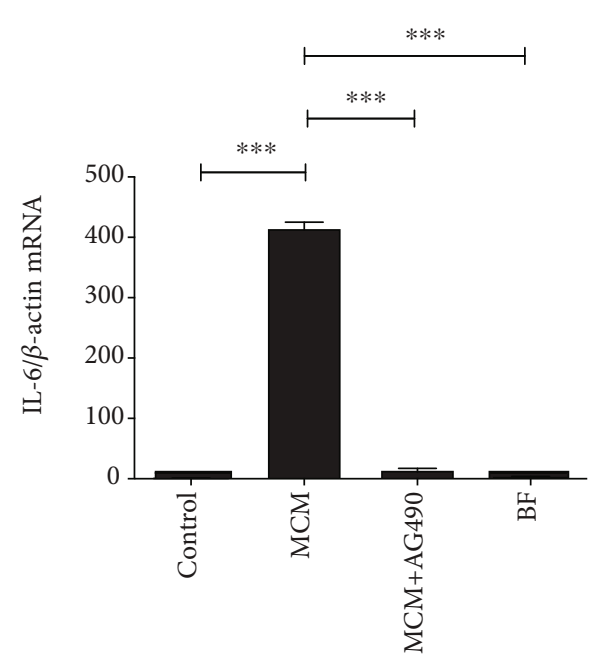

(c)

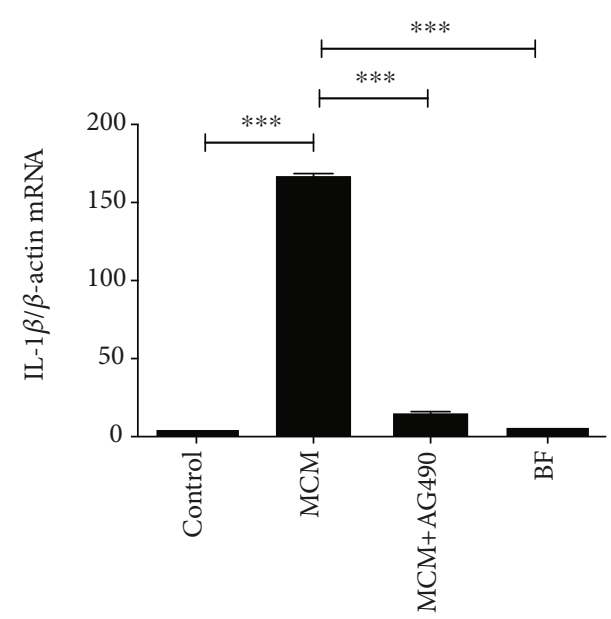

(e)

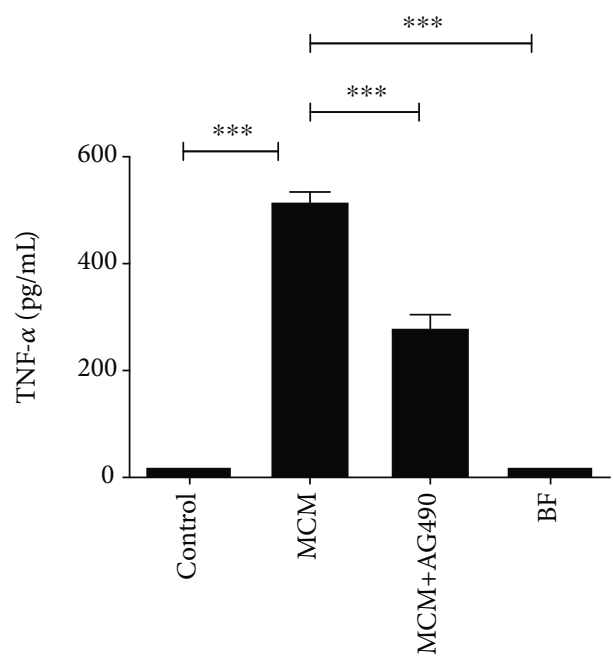

(b)

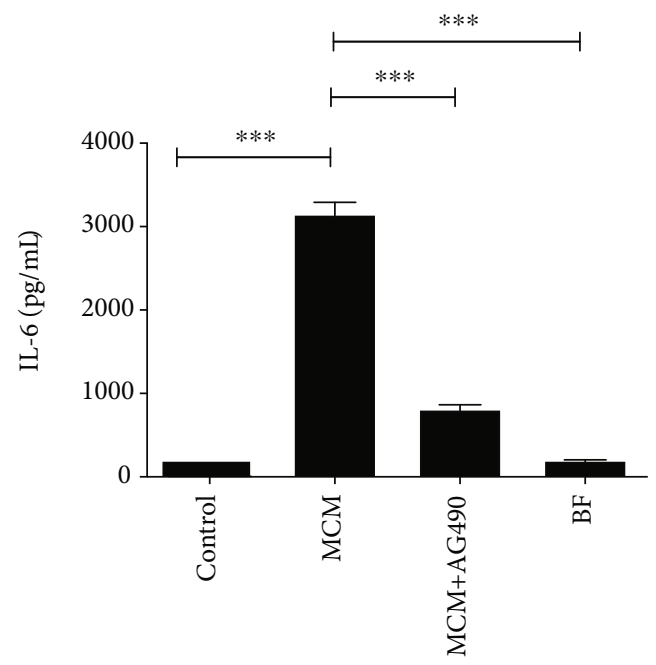

(d)

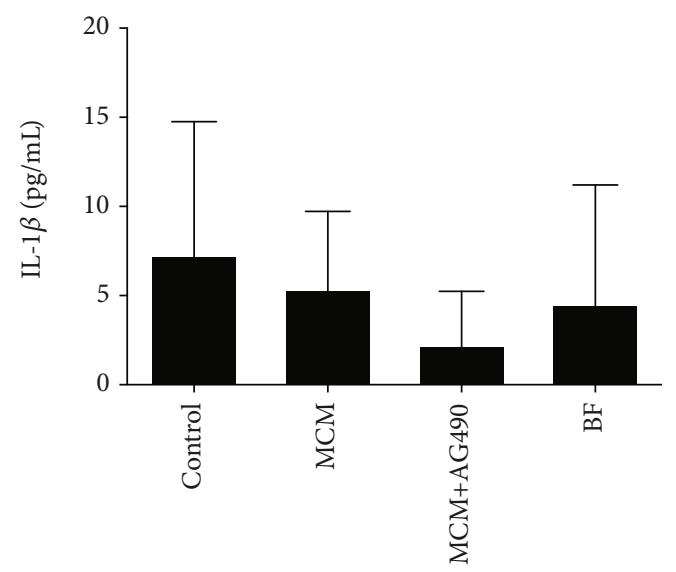

(f)

FIGURE 2: MCM increases the expression of proinflammatory mediators in leptomeningeal cells, which is suppressed by AG490. (a, b) TNF- $\alpha$ mRNA and protein levels in leptomeningeal cells after $12 \mathrm{~h}$ of treatment were measured by RT-qPCR and ELISA; (c, d) IL-6 mRNA and protein levels in leptomeningeal cells after $12 \mathrm{~h}$ of treatment were measured by RT-qPCR and ELISA; (e, f) IL-1 $\beta$ mRNA and protein levels in leptomeningeal cells after $12 \mathrm{~h}$ of treatment were measured by RT-qPCR and ELISA. Each column and bar represent the mean \pm SEM $(n=3$, each). Asterisks indicate a statistically significantly difference from the value in corresponding group $(* * P<0.01 ; * * * P<0.001)$. 


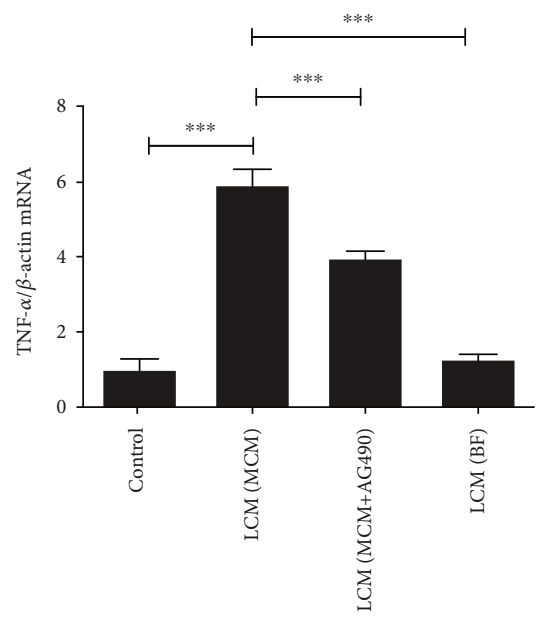

(a)

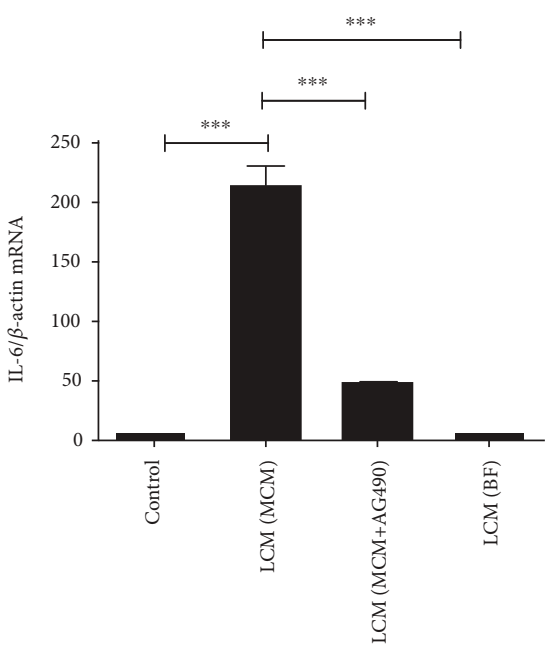

(c)

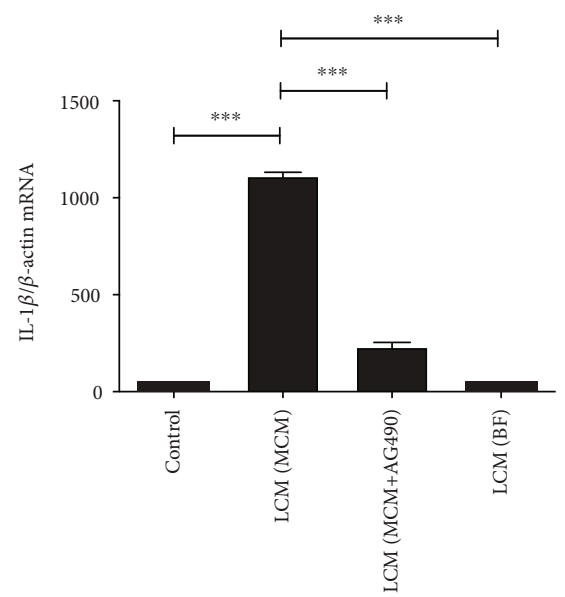

(e)

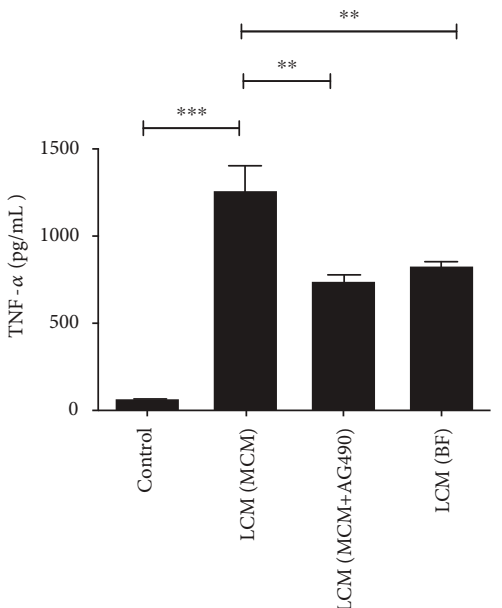

(b)

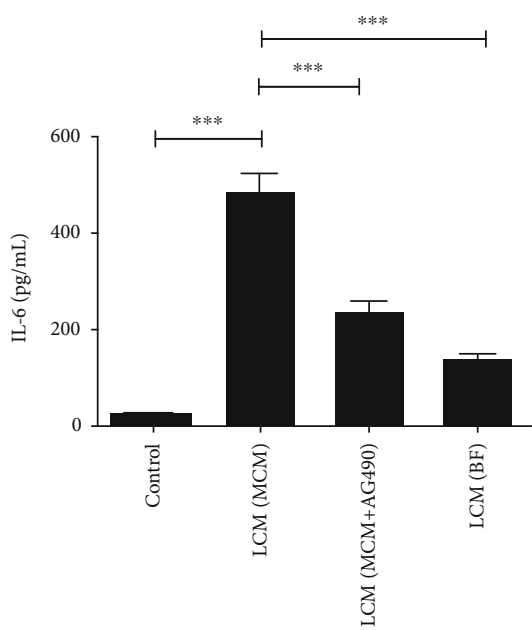

(d)

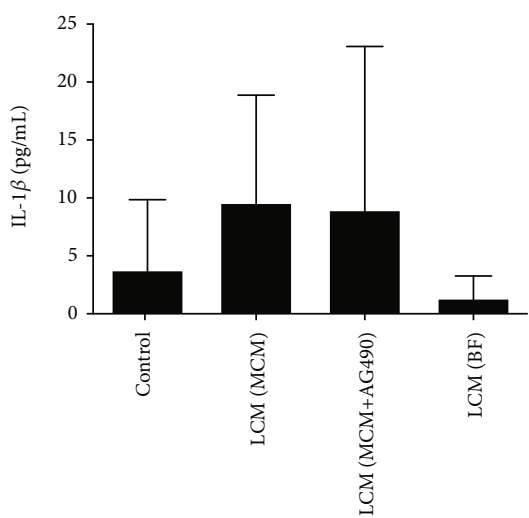

(f)

FIGURE 3: The effect of different LCMs on the expression of proinflammatory mediators in microglia. (a, b) TNF- $\alpha$ mRNA and protein levels in microglia after $24 \mathrm{~h}$ of incubation with LCMs or complete medium; (c, d) IL-6 mRNA and protein levels in microglia after $24 \mathrm{~h}$ of incubation with LCMs or complete medium; (e, f) IL- $1 \beta$ mRNA and protein levels in microglia after $24 \mathrm{~h}$ of incubation with LCMs or complete medium. Each column and bar represent the mean \pm SEM $(n=3$, each). Asterisks indicate a statistically significantly difference from the value in corresponding group $(* * P<0.01 ; * * * P<0.001)$. 

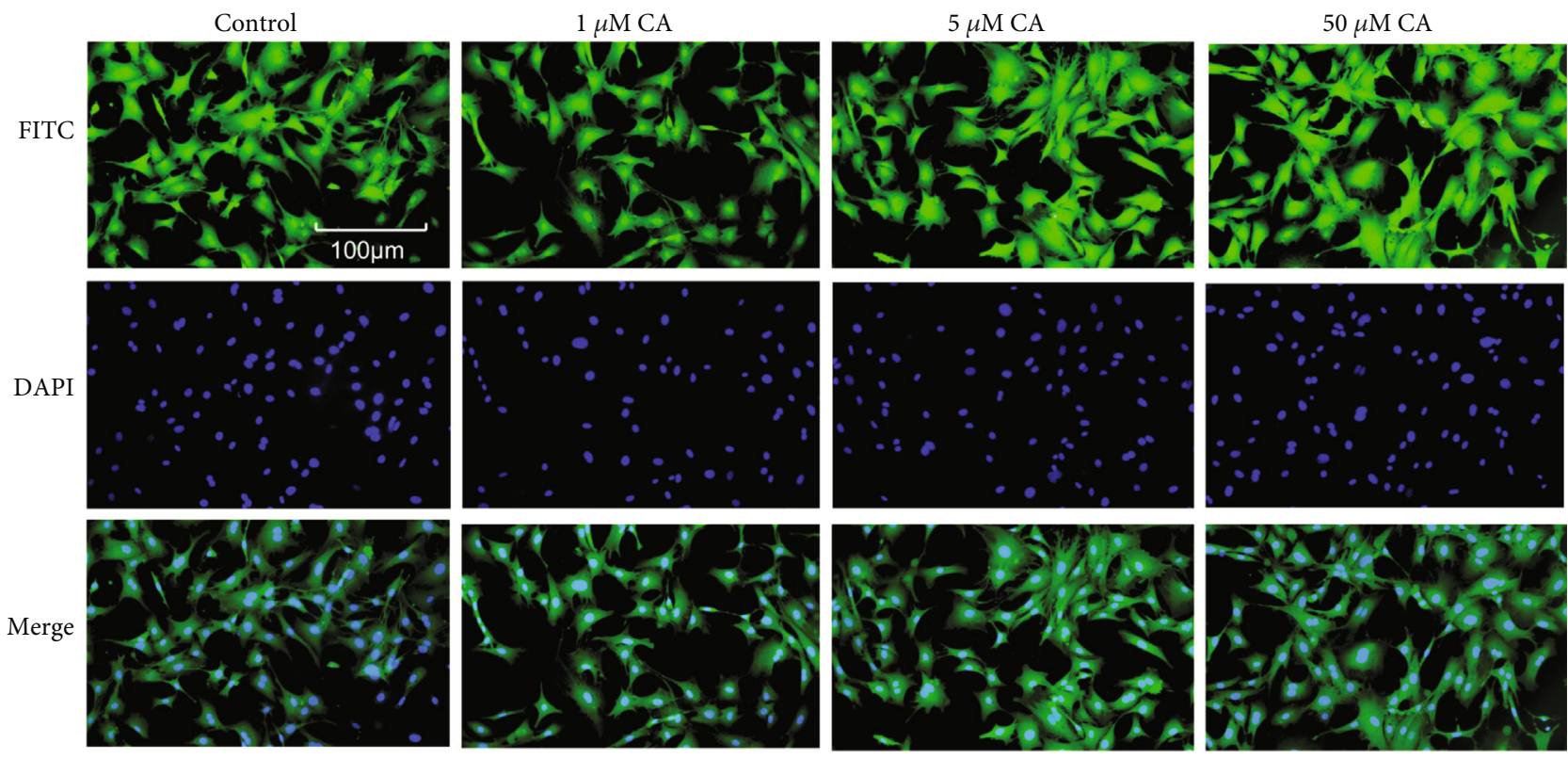

(a)

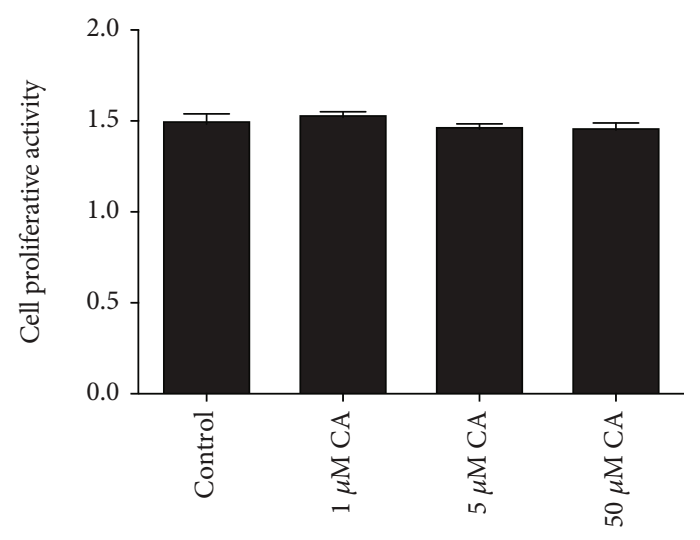

(b)

Figure 4: The effect of CA on morphology and proliferation of leptomeningeal cells was negligible. (a) Fluorescent staining under a microscope. Green represented cytoplasmic proteins stained with FITC, and blue represented the nuclei stained with DAPI. (b) CCK8 assay evaluated that the proliferation rates in leptomeningeal cells after $24 \mathrm{~h}$ of 1,5 , and $50 \mu \mathrm{M}$ CA treatment. Each column and bar represent the mean \pm SEM $(n=3$, each). There was no significant difference between groups.

compared with the MCM group. There were significant differences between the groups.

\section{Discussion}

Although LPS and proinflammatory factors as large molecules cannot easily penetrate the BBB, many clinical studies [30-32] have pointed out a potential link between chronic $\mathrm{PD}$, a common chronic systemic inflammation, and Alzheimer's disease $(\mathrm{AD})$. Evidences suggest that the presence of systemic inflammation may predate $\beta$-amyloid deposition, which is considered as the cause of $\mathrm{AD}$ [33]. This indicates that therapy aiming at reducing inflammatory responses may contribute to mild cognitive impairment such as $\mathrm{AD}$ [34]. Though these efforts focusing on the need to ameliorate central inflammation have been disappointing, little attention has been paid to the importance of dampening down systemic inflammation. The further exploration about the communication between the periphery and the brain is clearly warranted. What we did in our experiment was to prove the ability of leptomeningeal cells to transmit the inflammatory signals coming from macrophages to microglia. The underlying mechanism and the possible therapies were also revealed in our in vitro experiment.

It is reported that activated macrophages are polarized to two major phenotypes [35]. M1 macrophages have proinflammatory function that increases expressions of TNF- $\alpha$, Il-1 $\beta$, IL-6, HLA-DR, and inducible nitric oxide synthase (iNOS), and M2 macrophages express transforming growth factor (TGF- $\beta$ ) and IL-10 to play an anti-inflammatory role [36-38]. P. gingivalis LPS promotes M1 macrophage polarization. But current researches indicate that gingipains, pili, and capsule all involve in chronic PD [31, 39]. P. gingivalis as anaerobic bacteria lives in the gingival crevicular fluid that 


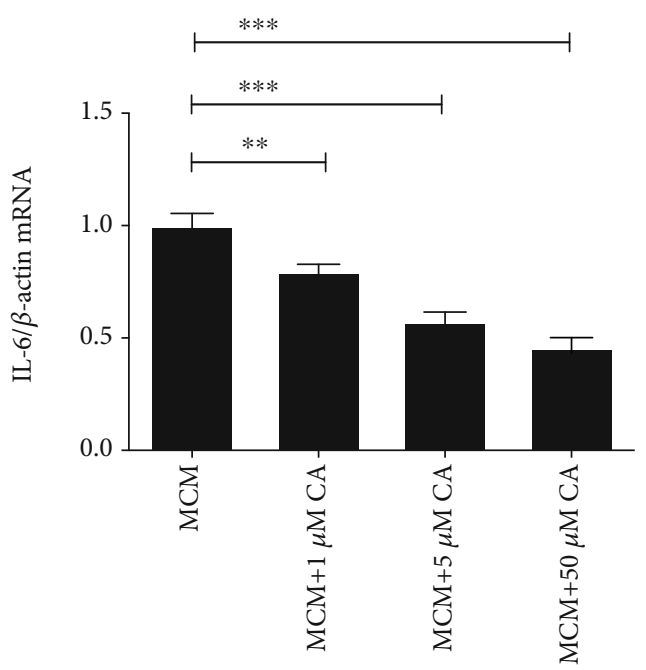

Figure 5: Inhibitory effects of AG490 on the expression of IL-6 mRNA in leptomeningeal cells treated with MCM. The IL-6 mRNA levels in leptomeningeal cells after $12 \mathrm{~h}$ of treatment were measured with RT-qPCR. Each column and bar represent the mean $\pm \operatorname{SEM}(n=3$, each). Asterisks indicate a statistically significantly difference from the value in corresponding group $(* * P<0.01 ; * * * P<0.001)$.

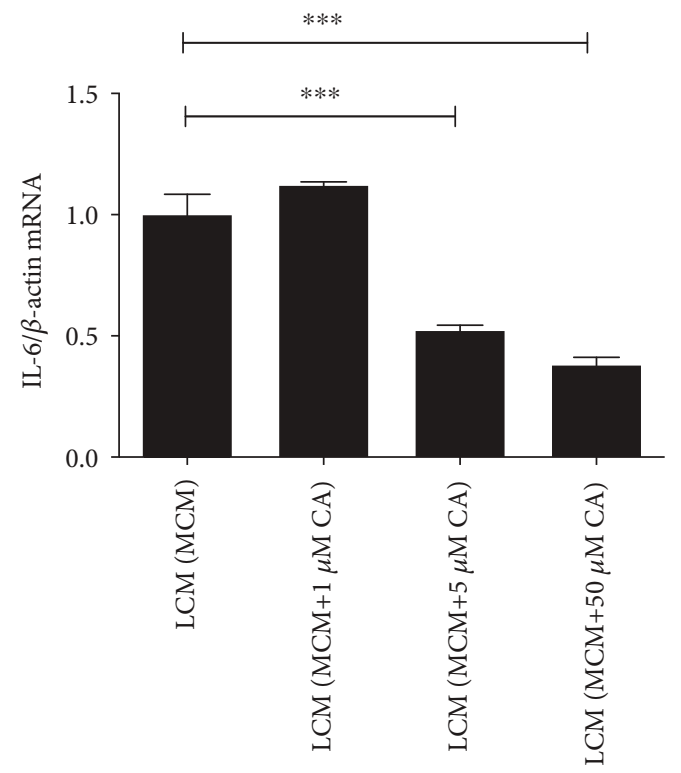

FIGURE 6: The effects of different LCMs on the expression of IL-6 mRNA in microglia. The IL-6 mRNA levels in microglia after $24 \mathrm{~h}$ of treatment were measured with RT-qPCR. Each column and bar represent the mean $\pm \operatorname{SEM}(n=3$, each). Asterisks indicate a statistically significantly difference from the value in corresponding group $(* * * P<0.001)$.

directly contacts oxygen. Its effect on macrophages in oxygen-rich condition is complicated and rarely reported.

We treated mouse macrophages with $P$. gingivalis or LPS. As we expected, $P$. gingivalis stimulated the release of TNF- $\alpha$ and IL- 6 . This indicates that $P$. gingivalis activates mouse macrophages to express proinflammatory factors even in an aerobic environment. Furthermore, this stimulation is different from that induced by LPS. IL-6 is more sensitive to bacteria than LPS. But TNF- $\alpha$ is quite the opposite. That means actual proportions of cytokines expressed in human bodies can be different from the results from a laboratory.

Some researchers also showed that JAK2/STAT participates in immune responses in activated macrophages induced by Staphylococcus aureus and LPS [40, 41]. Our data also implied that the JAK2-specific inhibitor, AG490 $(50 \mu \mathrm{g} / \mathrm{mL})$, downregulated expression levels of proinflammatory factors TNF- $\alpha$ and IL- 6 in macrophages treated with P. gingivalis (Figure 1). This is the first report that shows a JAK2 inhibitor suppresses the proinflammatory effect of macrophages induced by $P$. gingivalis. It points out a new direction to reduce the level of systemic inflammation in patients with $\mathrm{PD}$, but the exact mechanism is still not clear. It was reported that $P$. gingivalis induced the activation of the $\mathrm{CatB} / \mathrm{NF}-\kappa \mathrm{B}$ signaling in inflammatory macrophages [42]. Another research showed that LPS stimulated the expression of NF- $\kappa \mathrm{B}$ subunit p65 in human ciliary epithelial cells, resulting in the activation of receptor for growthhormone-releasing hormone (GHRH-R). The downstream JAK2 is phosphorylated and augments the production of proinflammatory factors [43]. Therefore, we speculate that JAK2 is likely to be a downstream molecular target of $\mathrm{CatB} / \mathrm{NF}-\kappa \mathrm{B}$ in the macrophage-mediated inflammatory reaction induced by $P$. gingivalis. Obviously, this theory needs to be validated by more studies.

MCM resulted in inflammatory responses in leptomeningeal cells (Figure 2). It is worthy of mentioning that MCM has been filtered to get rid of alive bacteria and cells but not excluding soluble bacterial products. So, we set the BF group and noticed the enhanced secretion of TNF- $\alpha$ and IL- 6 proteins, and mRNAs in the BF group were slightly stronger than that in the control group but significantly weaker than that in the MCM group. This indicates that there are the two components, proinflammatory factors from activated macrophages and soluble toxin from $P$. gingivalis, of MCM act on leptomeningeal cells, and proinflammatory factors have played a major role. In addition, the amount of IL- $1 \beta$ in the medium of all groups was so small that there was no significant difference between them (Figure 2(f)). Some bacterial metabolites can result in the ubiquitination and degradation of the inactive proform before mature IL- $1 \beta$ is produced [44]. This can be a probable explanation of this phenomenon. There is another possibility: except for primary stimulus like LPS, the secretion of IL- $1 \beta$ also depends on activators of the NLRP3 inflammasome, such as nigericin [45], and the activator can be deactivated in the aerobic medium. This speculation is also in need of verification.

Several inflammatory markers can be highly expressed in the serum of PD patients for a long time. Periodontal therapy did not seem to have any significant impact on the systemic cytokine levels [46]. That means leptomeningeal immune reaction will continue to persist in patients with chronic $\mathrm{PD}$, even if they are asymptomatic in the face of cognitive impairment. A leptomeningeal inflammation can lead to structural changes, damages to the physical barrier, and the entry of immune cells into the CNS [47]. No doubt it is 


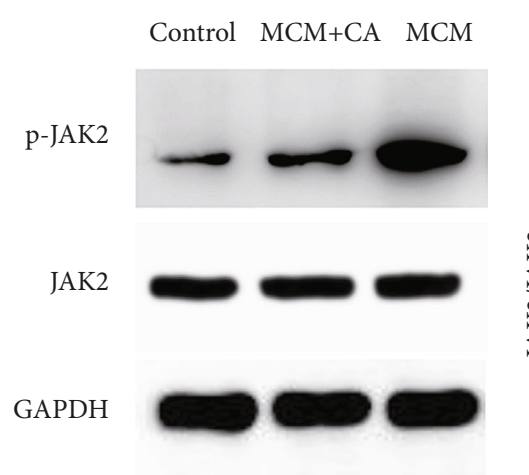

(a)

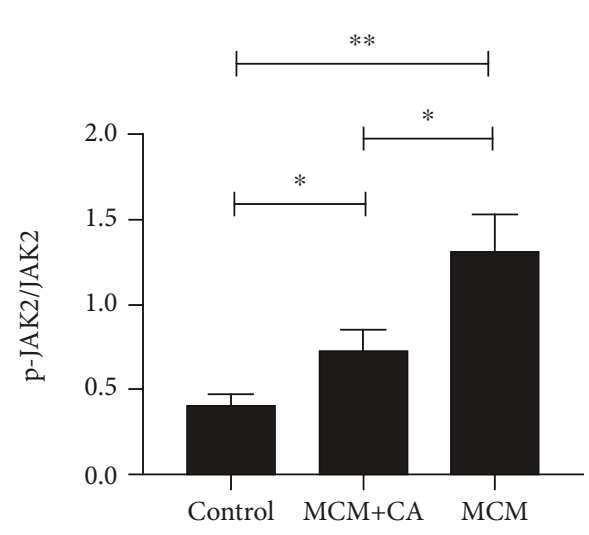

(b)

FIGURE 7: Inhibitory effect of CA on expressions of p-JAK2 protein in leptomeningeal cells activated by MCM. (a) The protein expressions of p-JAK2 and JAK2 in leptomeningeal cell were quantitated using western blot assays. (b) The quantitative analyses of the immunoblotting for p-JAK2/JAK2 in (a). Each column and bar represent the mean \pm SEM $(n=3$, each). Asterisks indicate a statistically significantly difference from the value in corresponding group $(* P<0.05 ; * * P<0.01)$.

associated with further neuroinflammation and neurodegenerative disease.

Geng et al. [48] and Xiong et al. [49] showed that inactivated JAK2 reduced inflammatory responses in cardiac muscle cells and macrophages, which was conductive to finding out a new target therapy against cardiac hypertrophy and insulin resistance. For AD, JAK2 can induce neuroinflammation via the activation of glial cells $[50,51]$, but the function of JAK2 can be opposite in spinal cord injury [52] and chemical nerve injury [53]. Meanwhile, JAK2 is involved in local inflammation in PD [54]. Allowing for the intricate role of JAK2 in diseases of the nervous system, identifying the exact effect of JAK2 on leptomeninges in PD is of vital importance.

We found that the p-JAK2/JAK2 expressions in leptomeningeal cells significantly increased after MCM incubation (Figure 7). p-JAK2 induces phosphorylated STAT3 to homodimerize and translocate to the nucleus, which is associated to angiotensin II, interferon- $\gamma$, IL- 6 , transforming growth factor- $\beta$ (TGF- $\beta$ ), iNOS, etc. $[55,56]$. By binding to its receptor, IL- 6 reactivates the JAK2 signaling in hepatic stellate cells (HSC) and upregulates itself, which forms a loop [57]. IL-6 was observed in MCM in our experiment (Figure 1), so we assumed that MCM could upregulate the proinflammatory factors of leptomeningeal cells by enhancing the JAK2 signaling. The obvious suppression of the inflammatory response induced by MCM was confirmed in leptomeningeal cells treated with AG490. This indicates that JAK2 is a novel target for therapy of inflammatory leptomeningeal reaction.

Furthermore, the medium coming from activated leptomeningeal cells triggered inflammatory activation of microglia, the key to neuroinflammation (Figure 3). This indicates that $P$. gingivalis in the peripheral system is able to cause CNS impairment and further neurodegenerative diseases. In a word, $P$. gingivalis induces macrophages to transmit inflammatory signals. Meanwhile, the JAK2-specific inhibitor blocks this process. These inflammatory signals subsequently activate leptomeningeal cells, initiating the immune response in microglia.
CA is a nontoxic compound extracted from natural plants. It is proved that CA can protect cells from radiation and neuroinflammation [58, 59]. Akram et al. [26] found the inhibitory effect of CA on paw edema of rat models induced by carrageenan (CRG). The effect on leptomeningeal cells has never been confirmed in the past reports. In our study, the CCK-8 assay and immunofluorescence analysis showed that the concentration of CA between 1 and $50 \mu \mathrm{M}$ did not affect the morphology or proliferation of leptomeningeal cells (Figure 4). So, it is proved that this concentration range is within a safe threshold to treat leptomeningeal cells. Immune responses in leptomeningeal cells induced by MCM were inhibited, and this inhibition was positively correlated with CA concentrations (Figure 5). This coincides with the decline in the inflammatory response in microglia activated by LCMs (Figure 6). This indicates a reduction in transmitting inflammation signals by $\mathrm{CA}$, which has been confirmed in vitro. Besides, CA effectively suppressed the upregulations of p-JAK2/JAK2 in leptomeningeal cells treated with MCM (Figure 7). This suggests that the effect of CA on JAK2 phosphorylation is involved in this reduction. We will provide more precise verification in a timely manner in a subsequent publication.

\section{Conclusions}

$P$. gingivalis bacteria are able to induce the obvious inflammatory response in macrophages, and this induction can be reduced by JAK2 inhibitor. The cytokines coming from macrophages activate leptomeningeal cells, accompanied by upregulated the activities of JAK2 in leptomeningeal cells. Inflammatory activation of leptomeningeal cells can transfer immune signals to microglia cells and trigger a series of inflammatory responses in the CNS. CA can downregulate the levels of inflammatory cytokines in leptomeninges via suppressing JAK2 phosphorylation, which reduces the immune response in microglia and the CNS. That means CA may be the next generation of drugs for many neurodegenerative diseases. 


\section{Data Availability}

The figures and table data used to support the findings of this study are included within the article.

\section{Ethical Approval}

The animal used in this study was approved by the Jilin University Animal Care and Use Committee. The animal experiments were carried out in accordance with requirements of the Experimental Animal Ethics and Welfare guidelines.

\section{Conflicts of Interest}

The authors declare no conflicts of interest.

\section{Acknowledgments}

This work was supported by funding from the National Natural Science Foundation of China to Yanmin Zhou (No. 81570983).

\section{References}

[1] G. Hajishengallis, T. Kajikawa, E. Hajishengallis et al., "Complement-dependent mechanisms and interventions in periodontal disease," Frontiers in Immunology, vol. 10, p. 406, 2019.

[2] P. I. Eke, B. A. Dye, L. Wei et al., "Update on prevalence of periodontitis in adults in the United States: NHANES 2009 to 2012," Journal of Periodontology, vol. 86, no. 5, pp. 611$622,2015$.

[3] A. El-Awady, M. de Sousa Rabelo, M. M. Meghil et al., "Polymicrobial synergy within oral biofilm promotes invasion of dendritic cells and survival of consortia members," $n p j$ Biofilms and Microbiomes, vol. 5, no. 1, 2019.

[4] G. Hajishengallis, "Periodontitis: from microbial immune subversion to systemic inflammation," Nature Reviews Immunology, vol. 15, no. 1, pp. 30-44, 2015.

[5] F. B. Teixeira, M. T. Saito, F. C. Matheus et al., "Periodontitis and Alzheimer's disease: a possible comorbidity between oral chronic inflammatory condition and neuroinflammation," Frontiers in Aging Neuroscience, vol. 9, 2017.

[6] S. Gaur and R. Agnihotri, "Alzheimer's disease and chronic periodontitis: is there an association?," Geriatrics \& Gerontology International, vol. 15, no. 4, pp. 391-404, 2015.

[7] Y. Liu, Z. Wu, X. Zhang et al., "Leptomeningeal cells transduce peripheral macrophages inflammatory signal to microglia in reponse toPorphyromonas gingivalisLPS," Mediators of Inflammation, vol. 2013, Article ID 407562, 11 pages, 2013.

[8] Z. Wu, J. Zhang, and H. Nakanishi, "Leptomeningeal cells activate microglia and astrocytes to induce IL-10 production by releasing pro-inflammatory cytokines during systemic inflammation," Journal of Neuroimmunology, vol. 167, no. 1-2, pp. 90-98, 2005.

[9] G. Kooij, K. Kopplin, R. Blasig et al., "Disturbed function of the blood-cerebrospinal fluid barrier aggravates neuro-inflammation," Acta Neuropathologica, vol. 128, no. 2, pp. 267-277, 2014.
[10] L. L. Muldoon, J. I. Alvarez, D. J. Begley et al., "Immunologic Privilege in the Central Nervous System and the Blood-Brain Barrier," Journal of Cerebral Blood Flow and Metabolism, vol. 33, no. 1, pp. 13-21, 2012.

[11] I. Decimo, G. Fumagalli, V. Berton, M. Krampera, and F. Bifari, "Meninges: from protective membrane to stem cell niche," American Journal Stem Cells, vol. 1, p. 105, 2012.

[12] K. Yasuda, C. Cline, P. Vogel et al., "Drug transporters on arachnoid barrier cells contribute to the blood-cerebrospinal fluid barrier," Drug Metabolism and Disposition, vol. 41, no. 4, pp. 923-931, 2013.

[13] H. B. Stolp, S. A. Liddelow, I. Sá-Pereira, K. M. Dziegielewska, and N. R. Saunders, "Immune responses at brain barriers and implications for brain development and neurological function in later life," Frontiers in Integrative Neuroscience, vol. 7, 2013.

[14] T. Wang, B. R. Wang, H. Z. Zhao et al., "Lipopolysaccharide up-regulates IL-6R $\alpha$ expression in cultured leptomeningeal cells via activation of ERK1/2 pathway," Neurochemical Research, vol. 33, no. 9, pp. 1901-1910, 2008.

[15] U.-K. Hanisch and H. Kettenmann, "Microglia: active sensor and versatile effector cells in the normal and pathologic brain," Nature Neuroscience, vol. 10, no. 11, pp. 1387-1394, 2007.

[16] M. T. Heneka, M. J. Carson, J. E. Khoury et al., "Neuroinflammation in Alzheimer's disease," The Lancet Neurology, vol. 14, no. 4, pp. 388-405, 2015.

[17] C. A. Mosser, S. Baptista, I. Arnoux, and E. Audinat, "Microglia in CNS development: shaping the brain for the future," Progress in Neurobiology, vol. 149-150, pp. 1-20, 2017.

[18] G. Pasqualetti, D. J. Brooks, and P. Edison, "The role of neuroinflammation in dementias," Current Neurology and Neuroscience Reports, vol. 15, no. 4, 2015.

[19] I. Morales, L. Guzm $\tilde{}_{i n} n-M a r t \tilde{A}-n e z$, C. Ã. ${ }^{3}$. Cerda-Troncoso, G. A. Far $\tilde{A}$-as, and R. B. Maccioni, "Neuroinflammation in the pathogenesis of Alzheimerâ $€^{\mathrm{TM}} \mathrm{s}$ disease. A rational framework for the search of novel therapeutic approaches," Frontiers in Cellular Neuroscience, vol. 8, 2014.

[20] S. Lattanzi, F. Brigo, E. Trinka, C. Cagnetti, M. di Napoli, and M. Silvestrini, "Neutrophil-to-lymphocyte ratio in acute cerebral hemorrhage: a system review," Translational Stroke Research, vol. 10, no. 2, pp. 137-145, 2019.

[21] Y. Zhou, Y. Wang, J. Wang, R. Anne Stetler, and Q. W. Yang, "Inflammation in intracerebral hemorrhage: from mechanisms to clinical translation," Progress in Neurobiology, vol. 115, pp. 25-44, 2014.

[22] J. Chen, X. Qu, Z. Li, D. Zhang, and L. Hou, "Peak neutrophilto-lymphocyte ratio correlates with clinical outcomes in patients with severe traumatic brain injury," Neurocritical Care, vol. 30, no. 2, pp. 334-339, 2019.

[23] P. L. McGeer and E. G. McGeer, "The amyloid cascadeinflammatory hypothesis of Alzheimer disease: implications for therapy," Acta Neuropathologica, vol. 126, no. 4, pp. 479497, 2013.

[24] D. Wang, J. Yin, R. Dong et al., "Inhibition of Janus kinase-2 signalling pathway ameliorates portal hypertensive syndrome in partial portal hypertensive and liver cirrhosis rats," Digestive and Liver Disease, vol. 47, no. 4, pp. 315-323, 2015.

[25] Y. Dong, T. Stewart, L. Bai et al., "Coniferaldehyde attenuates Alzheimer's pathology via activation of Nrf2 and its targets," Theranostics, vol. 10, no. 1, pp. 179-200, 2020.

[26] M. Akram, K. A. Kim, E. S. Kim et al., "Selective inhibition of JAK2/STAT1 signaling and iNOS expression mediates the 
anti-inflammatory effects of coniferyl aldehyde," Chemico-Biological Interactions, vol. 256, pp. 102-110, 2016.

[27] J. Jeon, H. Kwon, E. Cho et al., "The effect of coniferaldehyde on neurite outgrowth in neuroblastoma Neuro2a cells," Neurochemistry International, vol. 131, p. 104579, 2019.

[28] Y. Ohe, K. Ishikawa, Z. Itoh, and K. Tatemoto, "Cultured leptomeningeal cells secrete cerebrospinal fluid proteins," Journal of Neuroimmunology, vol. 67, no. 3, pp. 964-971, 1996.

[29] Z. Wu, Y. Hayashi, J. Zhang, and H. Nakanishi, "Involvement of prostaglandin E2 released from leptomeningeal cells in increased expression of transforming growth factor- $\beta$ in glial cells and cortical neurons during systemic inflammation," Journal of Neuroscience Research, vol. 85, no. 1, pp. 184-192, 2007.

[30] S. K. Singhrao and I. Olsen, "Assessing the role of Porphyromonas gingivalis in periodontitis to determine a causative relationship with Alzheimer's disease," Journal of Oral Microbiology, vol. 11, no. 1, p. 1563405, 2019.

[31] S. K. Singhrao and I. Olsen, "Are Porphyromonas gingivalis outer membrane vesicles microbullets for sporadic Alzheimer's disease manifestation?," Journal of Alzheimer's Disease Reports, vol. 2, no. 1, pp. 219-228, 2018.

[32] V. Ilievski, P. K. Zuchowska, S. J. Green et al., "Chronic oral application of a periodontal pathogen results in brain inflammation, neurodegeneration and amyloid beta production in wild type mice," Plos One, vol. 13, no. 10, article e0204941, 2018.

[33] R. J. O'Brien and P. C. Wong, "Amyloid precursor protein processing and Alzheimer's disease," Annual Review of Neuroscience, vol. 34, no. 1, pp. 185-204, 2011.

[34] C. Holmes, "Review: systemic inflammation and Alzheimer's disease," Neuropathology and Applied Neurobiology, vol. 39, no. 1, pp. 51-68, 2013.

[35] A. C. Trombetta, S. Soldano, P. Contini et al., "A circulating cell population showing both M1 and M2 monocyte/macrophage surface markers characterizes systemic sclerosis patients with lung involvement," Respiratory Research, vol. 19, no. 1, p. 186,2018

[36] D. Zhang, L. Chen, S. Li, Z. Gu, and J. Yan, "Lipopolysaccharide (LPS) ofPorphyromonas gingivalisinduces IL- $1 \beta$, TNF- $\alpha$ and IL- 6 production by THP-1 cells in a way different from that ofEscherichia coliLPS," Innate Immunity, vol. 14, no. 2 , pp. 99-107, 2008.

[37] S. Tedesco, C. Bolego, A. Toniolo et al., "Phenotypic activation and pharmacological outcomes of spontaneously differentiated human monocyte-derived macrophages," Immunobiology, vol. 220, no. 5, pp. 545-554, 2015.

[38] A. Shapouri-Moghaddam, S. Mohammadian, H. Vazini et al., "Macrophage plasticity, polarization, and function in health and disease," Journal of Cellular Physiology, vol. 233, no. 9, pp. 6425-6440, 2018.

[39] L. Jia, N. Han, J. du, L. Guo, Z. Luo, and Y. Liu, "Pathogenesis of important virulence factors of Porphyromonas gingivalis via toll-like receptors," Frontiers in Cellular and Infection Microbiology, vol. 9, pp. 262-262, 2019.

[40] A. Sun, H. Zhang, F. Pang et al., "Essential genes of the macrophage response to Staphylococcus aureus exposure," Cellular \& Molecular Biology Letters, vol. 23, no. 1, 2018.

[41] Y.-J. Kim, J. Lee, H.-J. Kim et al., “Anti-inflammatory effects of Angelica sinensis (Oliv.) diels water extract on RAW 264.7 induced with lipopolysaccharide," Nutrients, vol. 10, no. 5, p. 647, 2018.

[42] R. Nie, Z. Wu, J. Ni et al., "Porphyromonas gingivalis infection induces amyloid- $\beta$ accumulation in monocytes/macrophages," Journal of Alzheimer's disease : JAD, vol. 72, no. 2, pp. 479-494, 2019.

[43] W. C. Liang, J. L. Ren, Q. X. Yu et al., "Signaling mechanisms of growth hormone-releasing hormone receptor in LPSinduced acute ocular inflammation," Proceedings of the National Academy of Sciences, vol. 117, no. 11, pp. 60676074, 2020.

[44] D. Hancz, E. Westerlund, C. Valfridsson et al., "Streptolysin $\mathrm{O}$ induces the ubiquitination and degradation of Pro-IL$1 \beta$, Journal of Innate Immunity, vol. 11, no. 6, pp. 457468, 2019.

[45] A. Moeintaghavi, H. R. Arab, S. A. Rahim Rezaee et al., "The effects of smoking on expression of IL- 12 and IL- $1 \beta$ in gingival tissues of patients with chronic periodontitis," The Open Dentistry Journal, vol. 11, no. 1, pp. 595-602, 2017.

[46] A. Zekeridou, A. Mombelli, J. Cancela, D. Courvoisier, and C. Giannopoulou, "Systemic inflammatory burden and local inflammation in periodontitis: what is the link between inflammatory biomarkers in serum and gingival crevicular fluid?," Clinical and Experimental Dental Research, vol. 5, no. 2, pp. 128-135, 2019.

[47] B. Shrestha, D. Paul, and J. S. Pachter, "Alterations in tight junction protein and IgG permeability accompany leukocyte extravasation across the choroid plexus during neuroinflammation," Journal of Neuropathology \& Experimental Neurology, vol. 73, no. 11, pp. 1047-1061, 2014.

[48] Z. Geng, W. Y. Fan, B. Zhou et al., "FNDC5 attenuates obesityinduced cardiac hypertrophy by inactivating JAK2/STAT3associated inflammation and oxidative stress," Journal of Translational Medicine, vol. 17, no. 1, p. 107, 2019.

[49] X.-Q. Xiong, Z. Geng, B. Zhou et al., "FNDC5 attenuates adipose tissue inflammation and insulin resistance via AMPKmediated macrophage polarization in obesity," Metabolism, vol. 83, pp. 31-41, 2018.

[50] J. Wan, A. K. Y. Fu, F. C. F. Ip et al., “Tyk2/STAT3 signaling mediates beta-amyloid-induced neuronal cell death: implications in Alzheimer's disease," The Journal of Neuroscience, vol. 30, no. 20, pp. 6873-6881, 2010.

[51] K. Ceyzériat, L. B. Haim, A. Denizot et al., "Modulation of astrocyte reactivity improves functional deficits in mouse models of Alzheimer's disease," Acta Neuropathologica Communications, vol. 6, no. 1, p. 104, 2018.

[52] M. A. Anderson, J. E. Burda, Y. Ren et al., "Astrocyte scar formation aids central nervous system axon regeneration," Nature, vol. 532, no. 7598, pp. 195-200, 2016.

[53] J. P. O'Callaghan, K. A. Kelly, R. L. VanGilder, M. V. Sofroniew, and D. B. Miller, "Early activation of STAT3 regulates reactive astrogliosis induced by diverse forms of neurotoxicity," PLoS One, vol. 9, no. 7, article e102003, 2014.

[54] W. J. Bae, M. R. Shin, S. K. Kang et al., "HIF-2 inhibition supresses inflammatory responses and osteoclastic differentiation in human periodontal ligament cells," Journal of Cellular Biochemistry, vol. 116, no. 7, pp. 1241-1255, 2015.

[55] D. Kim, I. H. Lee, S. Kim et al., "A specific STAT3-binding peptide exerts antiproliferative effects and antitumor activity by inhibiting STAT3 phosphorylation and signaling," Cancer Research, vol. 74, no. 8, pp. 2144-2151, 2014. 
[56] R.-Y. Liu, Y. Zeng, Z. Lei et al., "JAK/STAT3 signaling is required for TGF- $\beta$-induced epithelial-mesenchymal transition in lung cancer cells," International Journal of Oncology, vol. 44, no. 5, pp. 1643-1651, 2014.

[57] W. Xu, P. Liu, and Y.-P. Mu, "Research progress on signaling pathways in cirrhotic portal hypertension," World Journal of Clinical Cases, vol. 6, no. 10, pp. 335-343, 2018.

[58] X. J. Li, K. W. Kim, H. Oh, X. Q. Liu, and Y. C. Kim, “Chemical Constituents and an Antineuroinflammatory Lignan, Savinin from the Roots ofAcanthopanax henryi," Evidence-Based Complementary and Alternative Medicine, vol. 2019, Article ID 1856294, 10 pages, 2019.

[59] S. Y. Kim, H. J. Lee, J. W. Nam, E. K. Seo, and Y. S. Lee, “Coniferyl aldehyde reduces radiation damage through increased protein stability of heat shock transcriptional factor 1 by phosphorylation," International Journal of Radiation Oncology • Biology • Physics, vol. 91, no. 4, pp. 807-816, 2015. 